\title{
Expression Profiling of LPS Responsive miRNA in Primary Human Macrophages
}

Afsar Raza Naqvi ${ }^{1}$, Sheng Zhong ${ }^{2}$, Hong Dang ${ }^{3}$, Jezrom B Fordham ${ }^{1}$, Salvador Nares ${ }^{1}$, Asma Khan ${ }^{2^{*}}$

${ }^{1}$ Department of Periodontics, College of Dentistry, University of Illinois at Chicago, USA

${ }^{2}$ Department of Endodontics, Center for Pain Research and Innovation, School of Dentistry, University of North Carolina, USA

${ }^{3}$ Cystic Fibrosis/Pulmonary Research and Treatment Center, Marisco Lung Institute, University of North Carolina, USA

\begin{abstract}
microRNAs (miRNAs) have emerged as important regulators of the innate and adaptive immune response. The purpose of the present study was to interrogate miRNA profiles of primary human macrophages challenged with bacterial lipopolysaccharide (LPS) with focus on expression kinetics. We employed Nanostring platform to precisely characterize the changes in miRNA expression following different doses and durations of LPS exposure. Differentially expressed miRNAs were identified in response to LPS challenge with convergent and divergent expression profiles. Pathway analysis of LPS-responsive miRNAs revealed regulation of biological processes linked to key cell signaling (including PIK3-Akt, MAP kinase, ErbB) and pathogen response pathways. Our data provide a comprehensive miRNA profiling of human primary macrophages treated with LPS. These results show that bacterial Toll like receptor (TLR) ligands can temporally modulate macrophage miRNA expression.
\end{abstract}

Keywords: microRNA; Lipopolysaccharide; Toll like receptor; Macrophages; Inflammation

\section{Introduction}

The initial immune response to bacterial infection involves the recognition of conserved pathogen-associated molecular patterns (PAMPs) such as LPS, lipoteichoic acid and peptidoglycans by pathogen recognition receptors (PRRs) including toll-like receptors (TLRs), RIGI-like receptors and NOD-like receptors [1-3]. PRRs are expressed by a variety of cell types including macrophages $(M \varphi)$, dendritic cells (DC), epithelial cells, fibroblasts and neutrophils $[1,4]$. Recognition of PAMPs initiates intracellular signaling cascades which results in the expression of inflammatory mediators with the aim of eliminating the invading microbes. Most microbial pathogens contain multiple PAMPs which are recognized by different PRRs. Furthermore, the same PAMP may activate different PRRs. Thus the host immune response has the ability to coordinate both cell-type specific as well pathogen-specific responses.

The human genome encodes 10 different TLRs (TLR1-10; 4,5). TLRs differ in their cellular localization, ligand specificity and adaptor molecules. TLR 1, 2, 4, 5 and 6 are present on the cell surface while, TLR $3,7,8,9$, and 10 are localized on endolysosomal membranes [4]. Each TLR can recognize specific ligands that will trigger unique immune response. Some TLRs can bind wide range of ligands (both foreign and host) but others have restricted ligand specificity (either foreign or host). For instance, TLR4 can bind LPS, heat shock proteins (HSPs), polysaccharides, viral proteins, etc., while TLR3 and TLR5 specifically recognizes double stranded RNA and bacterial flagellin, respectively $[1,5]$. Further, TLRs also differ in their requirement of adaptor molecule for signaling.

The outcome of TLR signaling is to prime cells for immune responses. This is primarily achieved by activation/repression of a large array of genes and microRNAs (miRNAs). The discovery of miRNAs has greatly expanded our understanding of the mechanisms that regulate gene expression. miRNAs are small (18-22 nucleotides), single stranded, noncoding RNA oligonucleotides. They regulate gene expression by binding to the 3'-UTR of target messenger RNAs (mRNAs) to down-regulate gene expression at the post-transcriptional level either by translational repression or mRNA degradation [6-8] More than 2500 miRNAs are encoded in the human genome and they regulate a wide variety of biological processes including proliferation, differentiation, cell fate determination, apoptosis, signal transduction and others [6,7]. A growing body of evidence supports the key role of miRNAs in the activation of both the innate and adaptive immune response [9-11]. Studies have shown changes in miRNA expression following LPS stimulation in various types of immune cells viz., monocytes, macrophages, etc. Several early and late LPS responsive miRNAs have been reported including miR-146, miR-155, members of let-7 family, etc. These miRNAs serve as positive and negative regulators of TLR signaling. They achieve this by targeting the components of TLR signaling pathways. For instance, miR-146 downregulates TRAF6, IRAK1 and IRAK2 while, let-7i and miR-105 target TLR4 and TLR2, respectively [12-15]. Therefore, changes in miRNA expression are not only part of host response to LPS, but also function to precisely control this response. Thus, identification of miRNA repertoire responsive to LPS challenge will be crucial in various bacterial infections.

In this report, CD14+ human macrophages were challenged with TLR4 agonist $E$. coli LPS, a widely accepted model to examine the innate response to microbial infection [16], and miRNAs were measured by the Nanostring nCounter platform. NanoString nCounter has been shown to be a novel, effective alternative to SYBR green real-time PCR $[17,18]$. It provides discrete counts of RNA transcripts and is capable of a high level of precision and sensitivity at less than one transcript copy per cell. By providing discrete counts of RNA transcripts, the

*Corresponding author: Asma A Khan, Department of Endodontics, Center for Pain Research and Innovation, School of Dentistry, University of North Carolina, USA, Tel: (919) 966 2707; E-mail: asma_khan@unc.edu

Received February 19, 2016; Accepted March 01, 2016; Published March 10 2016

Citation: Naqvi AR, Zhong S, Dang H, Fordham JB, Nares S, et al. (2016) Expression Profiling of LPS Responsive miRNA in Primary Human Macrophages. J Microb Biochem Technol 8: 136-143. doi: 10.4172/1948-5948.1000276

Copyright: @ 2016 Naqvi AR, et al. This is an open-access article distributed unde the terms of the Creative Commons Attribution License, which permits unrestricted use, distribution, and reproduction in any medium, provided the original author and source are credited. 
nCounter overcomes the saturation limitations of microarrays while avoiding the complex sequence analysis necessitated by RNA-Seq. The data gained provides precise and a real time changes in miRNA copy number and is highly relevant in clinical settings as demonstrated by various studies $[19,20]$. Here we demonstrate that miRNA expression in primary human macrophages is altered in time and dose-dependent manner upon E.coli LPS challenge. These miRNAs were predicted to target important signaling pathways.

\section{Materials and Methods}

\section{Primary human macrophages culture and LPS stimulation}

Freshly prepared buffy coats were collected from healthy donors ( $\mathrm{n}=3$; Sylvan N. Goldman Oklahoma Blood Institute, Oklahoma City, OK) by density gradient centrifugation, as described earlier [2125]. Briefly, PMBCs were purified using Ficoll Paque (GE Healthcare, Piscataway, NJ) based density centrifugation. PBMCs were incubated with magnetic-labeled CD14 beads (Miltenyi Biotech, Cologne, Germany), according to manufacturer's instructions. The purity of CD14+ cells was $95 \%$, as determined by flow cytometry. Monocytes were plated at $2 \times 10^{6} / \mathrm{ml}$ in DMEM supplemented with penicillin (100 $\mathrm{U} / \mathrm{ml})$ and streptomycin $(100 \mathrm{mg} / \mathrm{ml})$. After $2 \mathrm{~h}$, media was removed and replaced with media containing 10\% FBS (Life Technologies, Grand Island, NY) and $50 \mathrm{ng} / \mathrm{ml} \mathrm{rhM-CSF} \mathrm{(PeproTech,} \mathrm{Rocky} \mathrm{Hill,} \mathrm{NJ)} \mathrm{for} \mathrm{M} \varphi$ differentiation. At day 7, cells were harvested, and surface expression of CD14, CD68, and HLA-DR was examined by flow cytometric analysis (data not shown). On day 7, primary $\mathrm{M} \varphi$ were stimulated by Escherichia coli 055:B5 LPS (Sigma, St. Louis, MO) at 1 and $10 \mathrm{ng} / \mathrm{ml}$ and incubated for 1 and 8 hours. Controls consisted of differentiated $M \varphi$ cultured in the absence of LPS.

\section{Cell viability assay}

Cell viability was determined using the CellTiter 96 AQueous Cell Proliferation Assay Kit (Promega, Madison, WI) as described by Naqvi et al. [25]. Briefly, LPS treated or untreated $M \varphi$ were incubated with MTS reagent for $2 \mathrm{~h}$ and absorbance at 490nm were collected on a 96-well plate reader (Victor ${ }^{3}$, Perkin Elmer, Waltham, Massachusetts, USA), according to manufacturer's instructions.

\section{Total RNA preparation}

At the end of the incubation period, cells were lysed and total RNA extracted using the miRNeasy Mini kit (Qiagen, Germantown, MD) according to manufacturer's instructions. Total RNA was quantified and integrity assessed using the Nanodrop (Life Technologies) and Bioanalyzer (Agilent Technologies, Santa Clara, California, USA), respectively All RNA samples were processed by the Genomics and Bioinformatics Core, Lineberger Comprehensive Cancer Center at University of North Carolina at Chapel Hill.

\section{nCounter miRNA expression assay}

miRNA profiling was performed using the Nanostring nCounter technology, a multiplexed, color-coded probe assay. One hundred nanograms of total RNA was used to generate cDNA libraries according to the manufacturer's instructions. Ligation reactions, purification and dilution of probes were performed according to the manufacturer's instructions (www.nanostring.com). Hybridization reactions were performed according to the manufacturer's instructions with $5 \mathrm{ml}$ of the five-fold diluted sample preparation reaction. All hybridization reactions were incubated at $65^{\circ} \mathrm{C}$ for a minimum of $18 \mathrm{~h}$. Hybridized probes were purified and counted, following the manufacturer's instructions. All samples were processed by the Genomics and Bioinformatics Core, Lineberger Comprehensive Cancer Center at University of North Carolina at Chapel Hill. Data Collection was carried out in the nCounter Digital Analyzer on the nCounter Prep Station and Digital Analyzer (NanoString Technologies, Seattle, WA, USA) at the Lineberger Comprehensive Cancer Center. For each assay, a high-density scan (600 fields of view) was performed. Digital images were processed and the barcode counts were tabulated in a comma separated value (CSV) format.

\section{Data analysis}

Nanostring nCounter expression counts were inspected manually and samples with low counts throughout all probes comparable to the negative controls were identified as outliers and excluded from further analysis. The count data were normalized using the Bioconductor package, NanoStringNorm, using geometric means of low variance probes to adjust for sample content, and quantile normalization after background subtraction. The normalized expression values were $\log 2$ transformed and probes that do not have values greater than 4.587876 (or mean +2 standard deviations of negative controls) in any of the samples were filtered out. Differential expression (DE) were analyzed by a 4-way ANOVA model with donor as random effect factor, LPS dose and time of treatment as fixed effect factors with dose and time interaction. The assay plate information was used as a surrogate for batch effect factor in the ANOVA model. Differentially expressed miRNAs were filtered at p-value $<0.05$ and fold change $>1.5$. DE miRNAs from different comparisons were pooled and the normalized $\log 2$ expression values were standardized across all DE miRNAs to mean of 0 and standard deviation of 1 , before hierarchical clustering. The statistical analysis and clustering were performed using Partek Genomics Suite v6.6 (St. Louis, MS, USA)

\section{Prediction of miRNA targeted pathways}

For each sample set, the LPS responsive miRNAs were uploaded onto DIANA-miRPath (http://diana.imis.athena-innovation.gr/DianaTools/ index.php? $r=$ mirpath/index) for pathway prediction. Since numerous predicted miRNA targets await validated, we selected DIANA-microTCDS algorithm which included predicted miRNA targets.

\section{Results}

\section{Macrophages respond to LPS by altering miRNA expression}

$\mathrm{M} \varphi s$ respond to PAMPs by secreting proinflammatory cytokines that subsequently trigger both innate and adaptive arms of immunity. We first confirmed that $1 \mathrm{ng} / \mathrm{ml}$ of LPS challenge is sufficient to induce secretion of proinflammatory cytokine TNFa (data not shown). We also confirmed that LPS treatment did not affect the cell viability. To examine the role of miRNAs in the early phases ( $1 \mathrm{~h}$ and $8 \mathrm{~h}$ ) of LPS stimulation, we interrogated global miRNA profile in primary human $\mathrm{M} \varphi s$. The time-kinetics of miRNA expression was analyzed by profiling 593 miRNAs using the Nanostring platform. We identified several miRNAs that were significantly altered upon LPS stimulation. Figure 1 shows a heat map of differentially expressed miRNAs across all samples. Interestingly, differentially expressed miRNAs were predominantly downregulated across all the dose and time points examined (Figure 2A). M $\varphi$ s challenged with $1 \mathrm{ng} / \mathrm{ml}$ E. coli LPS for 1 hour show altered expression of 41 miRNAs; of which 5 exhibit induced expression while 36 were downregulated (Figure 2A; Table 1A). At 8 h, we noted 44 differentially expressed miRNAs compared with unchallenged controls (Figure 2A, Table 1B). Among these, 6 miRNAs exhibit increased expression while 38 were downregulated. We then searched for a 
Citation: Naqvi AR, Zhong S, Dang H, Fordham JB, Nares S, et al. (2016) Expression Profiling of LPS Responsive miRNA in Primary Human Macrophages. J Microb Biochem Technol 8: 136-143. doi: 10.4172/1948-5948.1000276

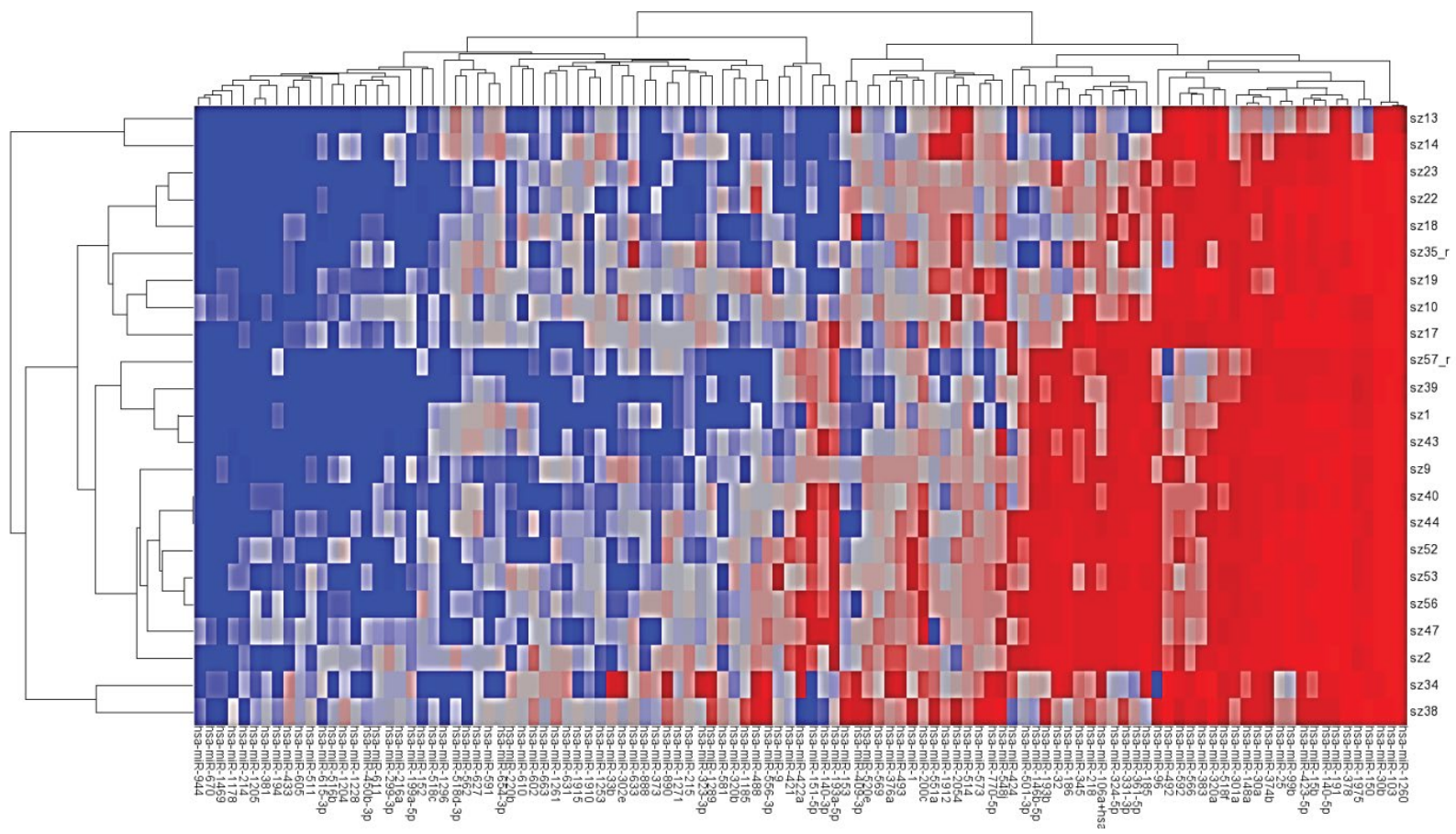

Figure 1: Heatmap showing miRNA expression in response to different doses and time points of LPS challenge. Samples are: Control\#17,47,11; 1ng/ $\mathrm{ml} ; 1 \mathrm{hr} \# 1,18,22,39,43 ; 1 \mathrm{ng} / \mathrm{ml} ; 8 \mathrm{hr} \# 9,13,52,56 ; 10 \mathrm{ng} / \mathrm{ml} ; 1 \mathrm{hr} \# 2,19,23,40,44 ; 10 \mathrm{ng} / \mathrm{ml} ; 8 \mathrm{hr} \#$ 10,14,53,57.

common set of differentially expressed miRNA, and found 11 miRNAs that were common to both the $1 \mathrm{~h}$ and $8 \mathrm{~h}$ time points (Figure $2 \mathrm{~B}, 2 \mathrm{C}$ ). The expression patterns of 10 of these (miR-146b, miR-193a, miR-193b, miR-218, miR-424, miR-450b-3p, miR-511, miR-573, miR-605 and miR-890) were similar at both time points. Our profiling identified previously reported LPS responsive miRNAs including miR-125b, miR146b, miR-200, 302a, and miR-511 [21-23]. Further, we also noticed altered expression of several miRNAs that were previously not reported including newly identified miRNAs such as miR-1178, miR-1261, miR1912 and miR-2054, etc., (Tables 1A and 1B). These results show LPS stimulation modulates $\mathrm{M} \varphi$ miRNA profiles temporally by altering expression of different subsets of miRNAs.

\section{Macrophages respond differentially to LPS dose}

Next we compared the impact of LPS dose on $\mathrm{M} \varphi$ miRNA profiles. To this end, M $\varphi$ s were challenged with $1 \mathrm{ng} / \mathrm{ml}$ and $10 \mathrm{ng} / \mathrm{ml}$ of $E$. coli LPS and miRNA expression was monitored at $1 \mathrm{~h}$ and $8 \mathrm{~h}$ post incubation. Analysis of $1 \mathrm{~h}$ samples showed 41 (5 up and 36 down) differentially expressed miRNAs with a challenge dose of $1 \mathrm{ng} / \mathrm{ml}$ LPS while at $10 \mathrm{ng} / \mathrm{ml}$ we identified 15 miRNAs (4 up and 11 down) with significant change in expression (Figure $2 \mathrm{~A}$ and Tables $1 \mathrm{~A}$ and $\mathrm{D}$ ). Among the differentially expressed miRNAs, 11 (miR-199a-5p, miR218, miR-409a, miR-433, miR-511, miR-514, miR-551, miR-562, miR556-3p, miR-1178 and miR-1205) were common to both doses (Figure 2D) and exhibited similar expression patterns (Tables $1 \mathrm{~A}$ and C).

The impact of LPS dose was also analyzed at $8 \mathrm{~h}$ post incubation. In cells treated with $10 \mathrm{ng} / \mathrm{ml}$ LPS, we noticed 63 differentially expressed miRNAs among which 17 were upregulated and 46 were downregulated (Figure 2A). These results suggest that $M \varphi$ stimulated for longer time periods and with higher LPS dose exhibit significant changes in miRNAs profiles. Interestingly, we also noticed that the prolonged stimulation of
$\mathrm{M} \varphi$ induced expression of greater numbers of miRNAs at this challenge dose (63 vs 44). Comparing miRNA profiles of cells challenged with 1 and $10 \mathrm{ng} / \mathrm{ml}$ LPS for $8 \mathrm{~h}$ we identified 28 miRNAs that were common to both doses (Figure 2E). Overall, our results indicate a correlation between miRNAs profiles and LPS dose in M $\varphi$.

\section{LPS responsive miRNAs are predicted to target key signaling and pathogen recognition pathways}

In order to investigate the impact of altered miRNA expression on biological output, we assessed the biological pathways and predicted targets of the LPS-responsive miRNAs. The miRNAs from each dataset were subjected to pathway analysis on DIANA miRPath v3.0 (http://snf-515788.vm.okeanos.grnet.gr/dianauniverse/index. php? $r=$ mirpath). From this we developed a list of predicted targets and biological pathways for the differentially expressed miRNAs. The list of 15 relevant pathways with significant p-values $(<0.01)$ for each dose and time response is provided in Table 2 . Several signaling pathways namely PIK3-Akt, MAPK, ErbB, Wnt and TGF- $\beta$ were highlighted. As expected, we noticed pathways involving pathogen recognition and clearance. These include endocytosis, bacterial invasion of cells and FcR-mediated phagocytosis. Polysaccharides and lipids metabolism, signaling and modification pathways were most commonly identified across all the datasets examined (Table 2A-D). These pathways are known to impact cell adhesion, motility, pathogen recognition and uptake.

Importantly, we observed a significant overlap in the pathways affected by miRNAs from different datasets indicating the role of miRNAs in polarizing the cellular responses. Nonetheless, analysis of miRNAs differentially expressed at higher LPS dose $(10 \mathrm{ng} / \mathrm{ml})$ revealed pathways not shared with lower dose $(1 \mathrm{ng} / \mathrm{ml})$ challenge at similar time point. Among these are two key pathways that include 


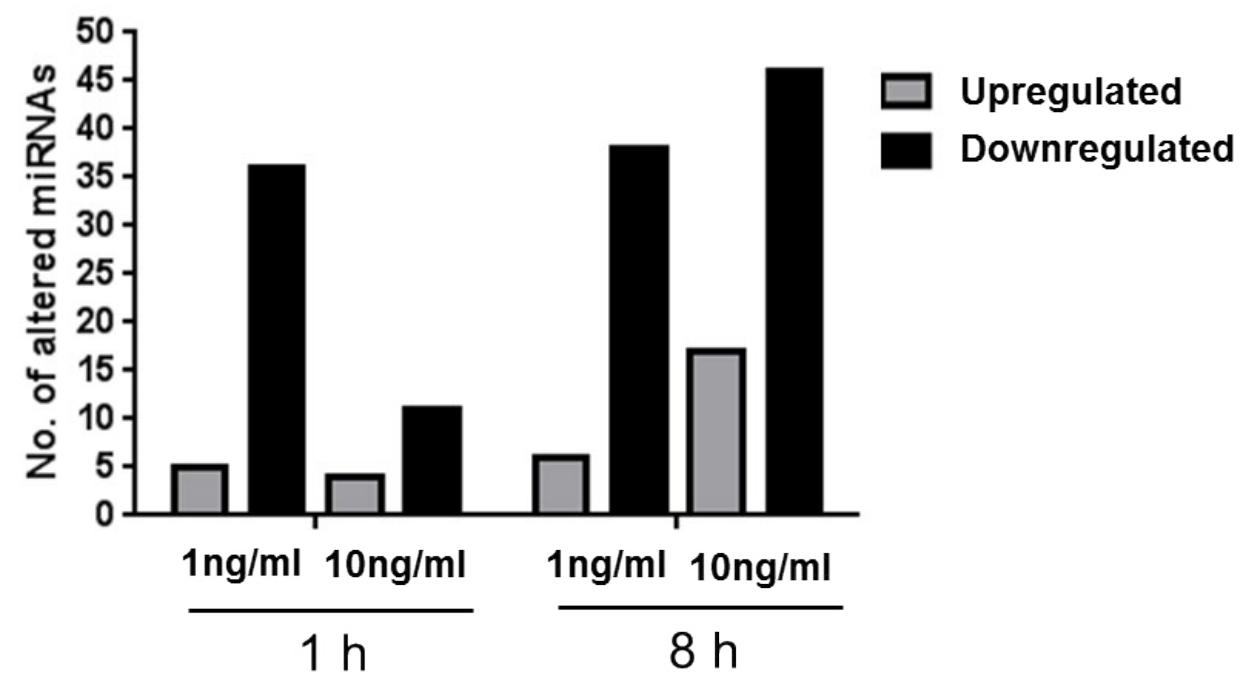

B

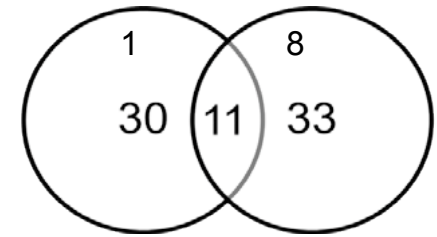

LPS $1 \mathrm{ng} / \mathrm{ml}$

C

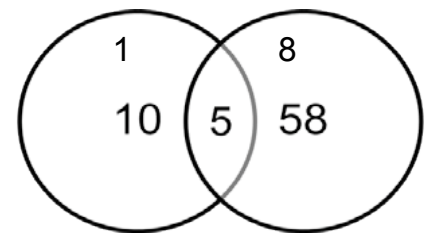

LPS $10 \mathrm{ng} / \mathrm{ml}$
D

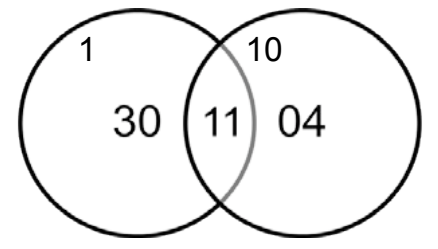

$1 \mathrm{~h}$

E

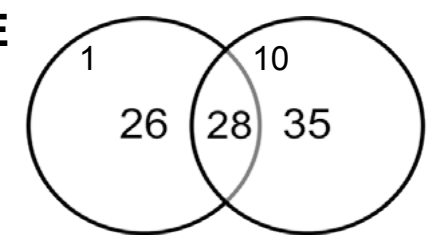

8

Figure 2 (A): Differential expression of miRNAs following LPS challenge at $1 \mathrm{ng} / \mathrm{ml}$ and $10 \mathrm{ng} / \mathrm{ml}$ for $1 \mathrm{~h}$ or 8 hours. (B-E): Common sets of differentially expressed miRNA following LPS challenge at specific doses and time points.

A. Human macrophages were treated with $1 \mathrm{ng} / \mathrm{ml}$ E.coli LPS for 1 hour.

\begin{tabular}{|c|c|c|}
\hline miRNA & Fold change & p-value \\
\hline hsa-miR-556-3p & -31.2343 & 0.000558 \\
\hline hsa-miR-890 & -27.2135 & $3.06 \mathrm{E}-05$ \\
\hline hsa-miR-199a-5p & -21.3307 & 0.002843 \\
\hline hsa-miR-1204 & -13.9828 & 0.03219 \\
\hline hsa-miR-488 & -12.1633 & 0.02769 \\
\hline hsa-miR-511 & -11.7723 & 0.010604 \\
\hline hsa-miR-302e & -11.3793 & 0.00766 \\
\hline hsa-miR-216a & -10.741 & 0.033464 \\
\hline hsa-miR-516b & -10.4053 & 0.015952 \\
\hline hsa-miR-409-3p & -10.0526 & 0.023857 \\
\hline hsa-miR-1261 & -9.57106 & 0.009325 \\
\hline hsa-miR-376a & -8.6461 & 0.003217 \\
\hline hsa-miR-33b & -8.18745 & 0.010264 \\
\hline hsa-miR-1253 & -7.87889 & 0.037582 \\
\hline hsa-miR-2052 & -7.77889 & 0.045247 \\
\hline hsa-miR-633 & -7.76623 & 0.021612 \\
\hline hsa-miR-605 & -7.68153 & 0.029125 \\
\hline hsa-miR-424 & -7.43781 & 0.020846 \\
\hline
\end{tabular}

\begin{tabular}{|c|c|c|}
\hline hsa-miR-770-5p & -7.01844 & 0.011542 \\
\hline hsa-miR-1205 & -6.95321 & 0.012067 \\
\hline hsa-miR-194 & -6.89172 & 0.032392 \\
\hline hsa-miR-569 & -6.82692 & 0.025647 \\
\hline hsa-miR-450b-3p & -6.64106 & 0.030425 \\
\hline hsa-miR-337-3p & -6.51625 & 0.043579 \\
\hline hsa-miR-548j & -6.47611 & 0.039445 \\
\hline hsa-miR-1912 & -6.17196 & 0.032997 \\
\hline hsa-miR-433 & -5.66386 & 0.031759 \\
\hline hsa-miR-1271 & -5.38944 & 0.004058 \\
\hline hsa-miR-193b & -5.17428 & 0.045688 \\
\hline hsa-miR-1178 & -4.85041 & 0.018656 \\
\hline hsa-miR-146b-5p & -4.62378 & 0.046233 \\
\hline hsa-miR-218 & -4.59623 & 0.035773 \\
\hline hsa-miR-320a & -3.59125 & 0.020025 \\
\hline hsa-miR-193a-5p & -3.5568 & 0.035694 \\
\hline hsa-miR-106a/17 & -3.24695 & 0.016031 \\
\hline hsa-miR-573 & -2.80172 & 0.045195 \\
\hline hsa-miR-514 & 2.50392 & 0.047209 \\
\hline hsa-miR-562 & 7.51529 & 0.025695 \\
\hline
\end{tabular}


Citation: Naqvi AR, Zhong S, Dang H, Fordham JB, Nares S, et al. (2016) Expression Profiling of LPS Responsive miRNA in Primary Human Macrophages. J Microb Biochem Technol 8: 136-143. doi: 10.4172/1948-5948.1000276

\begin{tabular}{|c|c|c|}
\hline hsa-miR-591 & 9.93073 & 0.026911 \\
\hline hsa-miR-551a & 11.1935 & 0.000599 \\
\hline hsa-miR-1296 & 17.3165 & 0.02919 \\
\hline
\end{tabular}

B. Human macrophages treated with $1 \mathrm{ng} / \mathrm{ml} \mathrm{E}$. coli LPS for 8 hours.

\begin{tabular}{|c|c|c|}
\hline miRNA & Fold change & p-value \\
\hline hsa-miR-193a-5p & -28.1509 & 0.000100164 \\
\hline hsa-miR-424 & -26.3899 & 0.0018661 \\
\hline hsa-miR-602 & -23.9685 & 0.0288658 \\
\hline hsa-miR-186 & -23.6563 & 0.0192091 \\
\hline hsa-miR-361-5p & -22.2274 & 0.0156237 \\
\hline hsa-miR-218 & -21.2598 & 0.000968802 \\
\hline hsa-miR-193b & -17.0989 & 0.00420067 \\
\hline hsa-miR-185 & -17.0196 & 0.0349622 \\
\hline hsa-miR-373 & -14.6641 & 0.0415649 \\
\hline hsa-miR-605 & -14.0025 & 0.0125141 \\
\hline hsa-miR-125b & -13.5135 & 0.0196724 \\
\hline hsa-miR-150 & -13.2099 & 0.00240754 \\
\hline hsa-miR-331-3p & -13.0433 & 0.00284728 \\
\hline hsa-miR-196b & -11.6311 & 0.0415904 \\
\hline hsa-miR-488 & -11.4972 & 0.0447984 \\
\hline hsa-miR-151-5p & -11.2514 & 0.0110432 \\
\hline hsa-miR-140-3p & -10.0222 & 0.0224999 \\
\hline hsa-miR-32 & -9.82883 & 0.0413303 \\
\hline hsa-miR-324-5p & -9.69977 & 0.00215447 \\
\hline hsa-miR-1975 & -9.63392 & 0.00459007 \\
\hline hsa-miR-450b-3p & -9.32785 & 0.0212821 \\
\hline hsa-miR-511 & -9.04912 & 0.0294384 \\
\hline hsa-miR-532-3p & -8.28998 & 0.0490303 \\
\hline hsa-miR-615-3p & -8.2303 & 0.0262171 \\
\hline hsa-miR-493 & -7.40798 & 0.0359485 \\
\hline hsa-miR-890 & -6.87393 & 0.00475192 \\
\hline hsa-miR-301a & -6.71513 & 0.0211866 \\
\hline hsa-miR-96 & -6.65422 & 0.045645 \\
\hline hsa-miR-146b-5p & -6.54631 & 0.0281515 \\
\hline hsa-miR-374b & -5.29633 & 0.0186839 \\
\hline hsa-miR-99b & -4.58917 & 0.0454594 \\
\hline hsa-miR-345 & -4.21347 & 0.0418053 \\
\hline hsa-miR-15b & -3.73893 & 0.00777077 \\
\hline hsa-miR-423-5p & -2.58407 & 0.044588 \\
\hline hsa-miR-200c & -2.50166 & 0.0441161 \\
\hline hsa-miR-1274b & -2.14825 & 0.00956674 \\
\hline hsa-miR-221 & -2.13986 & 0.00381351 \\
\hline hsa-miR-24 & -1.74958 & 0.020932 \\
\hline hsa-miR-514 & 2.88047 & 0.0379482 \\
\hline hsa-miR-573 & 3.10818 & 0.0438976 \\
\hline hsa-miR-518f & 3.30254 & 0.0304381 \\
\hline
\end{tabular}

C.Human macrophages treated with $10 \mathrm{ng} / \mathrm{ml}$ E. coli LPS for 1 hour.

\begin{tabular}{|c|c|c|}
\hline miRNA & Fold change & p-value \\
\hline hsa-miR-581 & -17.511 & 0.045932 \\
\hline hsa-miR-199a-5p & -9.06846 & 0.019608 \\
\hline hsa-miR-409-3p & -7.46168 & 0.043875 \\
\hline hsa-miR-153 & -6.914 & 0.041517 \\
\hline hsa-miR-518c & -5.97653 & 0.025675 \\
\hline hsa-miR-511 & -5.88614 & 0.050505 \\
\hline hsa-miR-1205 & -5.26955 & 0.026363 \\
\hline hsa-miR-556-3p & -5.16818 & 0.04638 \\
\hline
\end{tabular}

\begin{tabular}{|c|c|c|}
\hline hsa-miR-433 & -4.86279 & 0.046782 \\
\hline hsa-miR-1178 & -4.57924 & 0.022404 \\
\hline hsa-miR-218 & -4.09269 & 0.049502 \\
\hline hsa-miR-514 & 2.81549 & 0.028259 \\
\hline hsa-miR-562 & 9.75423 & 0.013981 \\
\hline hsa-miR-518d-3p & 11.0995 & 0.025983 \\
\hline hsa-miR-551a & 13.2433 & 0.000346 \\
\hline
\end{tabular}

D. Human macrophages treated with $10 \mathrm{ng} / \mathrm{ml}$ E. coli LPS for 8 hour.

\begin{tabular}{|c|c|c|}
\hline miRNA & Fold change & $p$-value \\
\hline hsa-miR-186 & 0.00212131 & -95.7265 \\
\hline hsa-miR-424 & 0.000360098 & -57.373 \\
\hline hsa-miR-29b & 0.00793683 & -45.433 \\
\hline hsa-miR-27a & 0.0194383 & -40.4845 \\
\hline hsa-miR-32 & 0.00307869 & -40.1821 \\
\hline hsa-miR-193a-5p & 4.56E-05 & -37.9784 \\
\hline hsa-miR-890 & 5.02E-05 & -30.7255 \\
\hline hsa-miR-891a & 0.0294375 & -30.0668 \\
\hline hsa-miR-193b & 0.00131651 & -28.6328 \\
\hline hsa-miR-151-5p & 0.00155507 & -26.6324 \\
\hline hsa-miR-331-3p & 0.000649021 & -23.0796 \\
\hline hsa-miR-19a & 0.0250877 & -22.7717 \\
\hline hsa-miR-532-3p & 0.00725674 & -22.5814 \\
\hline hsa-miR-185 & 0.0238436 & -21.8341 \\
\hline hsa-miR-18b & 0.0138967 & -14.7544 \\
\hline hsa-miR-423-3p & 0.0183292 & -13.8494 \\
\hline hsa-miR-140-3p & 0.0127852 & -13.1113 \\
\hline hsa-miR-218 & 0.005384 & -10.9014 \\
\hline hsa-miR-574-3p & 0.0178887 & -10.8706 \\
\hline hsa-miR-324-5p & 0.00167955 & -10.5147 \\
\hline $\begin{array}{l}\text { hsa-miR-520d-5p+hsa-miR- } \\
527+\text { hsa-miR-518a-5p }\end{array}$ & 0.0332644 & -10.447 \\
\hline hsa-miR-301a & 0.00822971 & -9.68532 \\
\hline hsa-miR-605 & 0.0279584 & -9.47062 \\
\hline hsa-miR-516a-5p & 0.0308221 & -9.39405 \\
\hline hsa-miR-496 & 0.0110786 & -9.2882 \\
\hline hsa-miR-31 & 0.0086838 & -9.28772 \\
\hline hsa-miR-150 & 0.0071677 & -8.85345 \\
\hline hsa-miR-146b-5p & 0.0164869 & -8.14325 \\
\hline hsa-miR-433 & 0.0299136 & -6.82542 \\
\hline hsa-miR-1975 & 0.0143885 & -6.44715 \\
\hline hsa-miR-107 & 0.0280177 & -5.66795 \\
\hline hsa-miR-1205 & 0.0420576 & -5.11632 \\
\hline hsa-miR-106a/17 & 0.00522892 & -4.78058 \\
\hline hsa-miR-188-5p & 0.0445391 & -4.3485 \\
\hline hsa-miR-374b & 0.0351116 & -4.29073 \\
\hline hsa-miR-15b & 0.00462133 & -4.19912 \\
\hline hsa-miR-345 & 0.0434297 & -4.15768 \\
\hline hsa-miR-376a & 0.0476912 & -4.12547 \\
\hline hsa-miR-532-5p & 0.027525 & -3.65767 \\
\hline hsa-miR-509-3p & 0.0326475 & -3.38043 \\
\hline hsa-miR-1260 & 0.0254564 & -2.94232 \\
\hline hsa-miR-30c & 0.0451624 & -2.83118 \\
\hline hsa-miR-22 & 0.0228818 & -2.76943 \\
\hline hsa-let-7a & 0.0243692 & -2.443 \\
\hline hsa-miR-221 & 0.00143387 & -2.40003 \\
\hline hsa-miR-1274b & 0.008082 & -2.19725 \\
\hline hsa-miR-764 & 0.0468539 & 3.29166 \\
\hline
\end{tabular}


Citation: Naqvi AR, Zhong S, Dang H, Fordham JB, Nares S, et al. (2016) Expression Profiling of LPS Responsive miRNA in Primary Human Macrophages. J Microb Biochem Technol 8: 136-143. doi: 10.4172/1948-5948.1000276

\begin{tabular}{|c|c|c|}
\hline hsa-miR-573 & 0.0348119 & 3.31514 \\
\hline hsa-miR-450b-5p & 0.0399014 & 3.68501 \\
\hline hsa-miR-1180 & 0.0134275 & 3.71005 \\
\hline hsa-miR-383 & 0.00835454 & 3.82831 \\
\hline hsa-miR-760 & 0.0359474 & 4.69097 \\
\hline hsa-miR-449a & 0.0445483 & 4.86847 \\
\hline hsa-miR-759 & 0.045053 & 4.934 \\
\hline hsa-miR-566 & 0.0181896 & 5.16446 \\
\hline hsa-miR-2054 & 0.0210186 & 7.09867 \\
\hline hsa-miR-432 & 0.0379926 & 7.3013 \\
\hline hsa-miR-551a & 0.00374997 & 7.78185 \\
\hline hsa-miR-631 & 0.0415932 & 12.3371 \\
\hline hsa-miR-924 & 0.0284851 & 13.7512 \\
\hline hsa-miR-644 & 0.0108704 & 17.7977 \\
\hline hsa-miR-1323 & 0.00598167 & 21.0441 \\
\hline hsa-miR-1915 & 0.00463882 & 21.5772 \\
\hline
\end{tabular}

Table 1: List of differentially expressed miRNAs responsive to E. coli LPS dose and time identified in primary human macrophages.

A. Predicted pathways of $M \varphi$ miRNAs responsive to $1 \mathrm{ng} / \mathrm{ml} E$. coli LPS challenged for $1 \mathrm{~h}$.

\begin{tabular}{|c|c|c|c|}
\hline KEGG pathway & p-value & \#genes & \#miRNAs \\
\hline Adherens junction & $9.68 \mathrm{E}-12$ & 59 & 30 \\
\hline Cell cycle & $6.11 \mathrm{E}-06$ & 82 & 31 \\
\hline TGF-beta signaling pathway & $6.67 \mathrm{E}-05$ & 51 & 30 \\
\hline ErbB signaling pathway & 0.000115 & 59 & 28 \\
\hline Endocytosis & 0.000192 & 123 & 31 \\
\hline Focal adhesion & 0.000192 & 129 & 32 \\
\hline p53 signaling pathway & 0.000258 & 50 & 27 \\
\hline Rap1 signaling pathway & 0.000259 & 127 & 30 \\
\hline PI3K-Akt signaling pathway & 0.000521 & 198 & 31 \\
\hline Regulation of actin cytoskeleton & 0.002879 & 124 & 29 \\
\hline mTOR signaling pathway & 0.003035 & 41 & 27 \\
\hline AMPK signaling pathway & 0.003339 & 78 & 29 \\
\hline ECM-receptor interaction & 0.006131 & 46 & 28 \\
\hline Fc gamma R-mediated phagocytosis & 0.035122 & 55 & 25 \\
\hline Ras signaling pathway & 0.035122 & 123 & 30 \\
\hline B. & & & \\
\hline
\end{tabular}

B. Predicted pathways of M $\varphi$ miRNAs responsive to $1 \mathrm{ng} / \mathrm{ml}$ E. coli LPS challenged for $8 \mathrm{~h}$.

\begin{tabular}{|c|c|c|c|}
\hline KEGG pathway & p-value & \#genes & \#miRNAs \\
\hline ErbB signaling pathway & $5.59 \mathrm{E}-06$ & 63 & 32 \\
\hline TGF-beta signaling pathway & $5.74 \mathrm{E}-06$ & 53 & 31 \\
\hline Endocytosis & $2.01 \mathrm{E}-05$ & 130 & 32 \\
\hline Ras signaling pathway & 0.000149 & 133 & 35 \\
\hline MAPK signaling pathway & 0.000745 & 151 & 37 \\
\hline Wnt signaling pathway & 0.001299 & 86 & 33 \\
\hline Regulation of actin cytoskeleton & 0.002783 & 125 & 32 \\
\hline Rap1 signaling pathway & 0.002783 & 124 & 34 \\
\hline Adherens junction & 0.014892 & 47 & 31 \\
\hline Focal adhesion & 0.014892 & 117 & 33 \\
\hline cGMP-PKG signaling pathway & 0.027851 & 93 & 34 \\
\hline AMPK signaling pathway & 0.027851 & 70 & 31 \\
\hline PI3K-Akt signaling pathway & 0.027851 & 182 & 34 \\
\hline SNARE interactions in vesicular transport & 0.029446 & 21 & 22 \\
\hline p53 signaling pathway & 0.039423 & 42 & 26 \\
\hline
\end{tabular}

C. Predicted pathways of $M \varphi$ miRNAs responsive to $10 \mathrm{ng} / \mathrm{ml} E$. coli LPS challenged for $1 \mathrm{~h}$.

\begin{tabular}{|c|c|c|c|}
\hline KEGG pathway & $p$-value & \#genes & \#miRNAs \\
\hline AMPK signaling pathway & 7.67E-05 & 42 & 10 \\
\hline mTOR signaling pathway & 0.000919 & 24 & 10 \\
\hline Phosphatidylinositol signaling system & 0.001834 & 24 & 9 \\
\hline TGF-beta signaling pathway & 0.008479 & 23 & 10 \\
\hline $\mathrm{N}$-Glycan biosynthesis & 0.005766 & 12 & 8 \\
\hline Adipocytokine signaling pathway & 0.019586 & 22 & 9 \\
\hline mRNA surveillance pathway & 0.020065 & 28 & 11 \\
\hline RNA degradation & 0.036259 & 27 & 10 \\
\hline $\begin{array}{l}\text { Glycosylphosphatidylinositol(GPI)-anchor } \\
\text { biosynthesis }\end{array}$ & 0.03644 & 9 & 6 \\
\hline Wnt signaling pathway & 0.03644 & 39 & 11 \\
\hline Gap junction & 0.037595 & 21 & 11 \\
\hline cGMP-PKG signaling pathway & 0.046164 & 44 & 10 \\
\hline Sphingolipid signaling pathway & 0.048088 & 31 & 10 \\
\hline $\begin{array}{l}\text { Glycosaminoglycan biosynthesis - keratan } \\
\text { sulfate }\end{array}$ & 0.048206 & 4 & 3 \\
\hline
\end{tabular}

D. Predicted pathways of $M \varphi$ miRNAs responsive to $10 \mathrm{ng} / \mathrm{ml}$ E. coli LPS challenged for $8 \mathrm{~h}$.

\begin{tabular}{|c|c|c|c|}
\hline KEGG pathway & p-value & \#genes & \#miRNAs \\
\hline ErbB signaling pathway & $1.35 \mathrm{E}-07$ & 73 & 44 \\
\hline ECM-receptor interaction & $4.50 \mathrm{E}-06$ & 55 & 39 \\
\hline Ras signaling pathway & $9.94 \mathrm{E}-06$ & 154 & 49 \\
\hline Focal adhesion & $2.26 \mathrm{E}-05$ & 148 & 48 \\
\hline Endocytosis & $6.10 \mathrm{E}-05$ & 147 & 47 \\
\hline TGF-beta signaling pathway & 0.000179 & 55 & 38 \\
\hline Mucin type O-Glycan biosynthesis & 0.000251 & 20 & 23 \\
\hline Rap1 signaling pathway & 0.000202 & 145 & 48 \\
\hline Adherens junction & 0.002025 & 56 & 43 \\
\hline Wnt signaling pathway & 0.000226 & 100 & 46 \\
\hline Viral carcinogenesis & 0.001341 & 118 & 47 \\
\hline Phosphatidylinositol signaling system & 0.002201 & 56 & 43 \\
\hline Bacterial invasion of epithelial cells & 0.00222 & 56 & 39 \\
\hline PI3K-Akt signaling pathway & 0.002786 & 217 & 48 \\
\hline MAPK signaling pathway & 0.002989 & 169 & 50 \\
\hline
\end{tabular}

Table 2: List of pathways predicted to be targeted by LPS responsive miRNAs. Only top 15 pathways are mentioned.

phosphatidylinositol signaling system and bacterial invasion of cells. Taken together, these results show that miRNA modulation can affect various cellular processes.

\section{Discussion}

The innate immune response to PAMPs is associated with modulation of the host cell transcriptome. LPS from various pathogens has been demonstrated to have profound impact on gene expression. Examining the regulation of such rapid changes in gene expression is key to elucidating the underlying mechanisms. miRNAs have emerged as potent regulators of mRNA stability and translation. Studies have shown involvement of several miRNAs in LPS treatment. However, the global miRNA profiles in primary human $M \varphi$ have not been previously comprehensively examined. Here we provide updated miRNA 
profiling of LPS-stimulated M $\varphi s$ and quantitated miRNA expression changes as a function of time and challenge dose. Our data shows that LPS challenge alters the miRNA profiles of primary human $\mathrm{M} \varphi$ and confirms differential expression of previously described miRNAs as well as miRNAs not reported in primary $\mathrm{M} \varphi$ using the Nanostring platform including a common set of LPS-responsive miRNAs. As our profiling included miRNAs from recent miRBase database (version 17), several novel miRNAs with potential roles in LPS signaling and/or response are identified. The role of these newly discovered miRNA is yet to be understood. Nonetheless, the changes in their expression would undoubtedly have an impact on their potential targets. Investigations on elucidating the functional role of these miRNAs in LPS treatment will uncover finer details of LPS signaling.

We observed different sets of altered miRNAs in a time and dose dependent manner. For instance, only 11 miRNAs were common to $1 \mathrm{~h}$ and $8 \mathrm{~h}$ samples when $\mathrm{M} \varphi$ were challenged with $1 \mathrm{ng} / \mathrm{ml} \mathrm{LPS}$ (Figure 2A) while 28 were shared between $1 \mathrm{ng} / \mathrm{ml}$ and $10 \mathrm{ng} / \mathrm{ml}$ LPS at $8 \mathrm{hr}$ (Figure 2C and D). Nonetheless, our pathway analysis revealed significant overlap of pathways affected by the miRNAs. For instance, PI3K-Akt pathway was commonly targeted by miRNAs. PI3K activation is key inhibitory pathway in limiting NFKB activity and therefore checks proinflammatory cytokine (e.g. TNF- $\alpha$, IL-6) production following LPS stimulation [26-29]. Several LPS responsive miRNAs target same pathway indicating regulatory fine tuning of these critical signaling pathways. Of the top 20 pathways, we noticed $\sim 15$ that were common for all the datasets. While a single miRNA can control expression of hundreds of target mRNA, several miRNAs can also bind and cooperatively regulate expression of the same transcript $[7,8]$. Taken together, these observations suggest that similar cellular functions are regulated by different sets of miRNAs.

Global profiling identified several novel LPS responsive miRNAs and also confirms miRNAs that are previously demonstrated to respond and regulate LPS signaling. For instance, miRNA profiling of monocytederived $M \varphi$ and DCs identified miR-511 as being differentially induced [23]. Interestingly, overexpression of miR-511 was shown to increase levels of its validated target TLR4, thereby acting as positive regulator of TLR expression in DCs. In the present study, we show that miR-511 is downregulated in response to LPS and its expression varies with the dose. While miR-511 expression is $\sim 12$ fold downregulated in cells treated with $1 \mathrm{ng} / \mathrm{ml} \mathrm{LPS}$, its expression is to $\sim 5$ folds at $10 \mathrm{ng} / \mathrm{ml} \mathrm{LPS}$ dose. TLR4 expression is known to increase with LPS stimulation [23]. Taken together, these observations suggest that high LPS dose increases expression of miR-511 and which in turn, may contribute to elevated TLR4 levels.

We noted several miRNAs that were responsive to LPS from E.coli and other gram-negative bacteria including the periodontal pathogens A. actinomycetemcomitans and $P$. gingivalis derived LPS [21]. While E.coli LPS is TLR4 agonist, $A$. actinomycetemcomitans and $P$. gingivalis LPS are TLR2 and TLR2/4 agonist, respectively. This indicates that LPS signaling emanating from different TLRs converge at the activation of common set of transcription factors regulating expression of these miRNAs. Our recent findings showed that miR-24 and miR-30 regulate phagocytosis of E. coli by myeloid inflammatory cells [25]. Both miR-24 and members of miR-30 were downregulated in E. coli LPS treated M $\varphi$ and this corroborates with our findings showing reduced expression of these miRNAs in E.coli challenged myeloid cells. Together, these observations indicate that highly similar impact on miRNA expression by $E$. coli LPS as well as whole bacteria which will have functional implication on downstream functions. Consistent with this, we noted that LPS responsive differentially expressed miRNAs were predicted to target pathways involved in pathogen recognition and uptake.

TLR4 signaling can be activated by either MyD (MyD-dependent) or TRIF (MyD-independent) adaptor proteins. MyD-dependent signaling culminates in the activation of NFKB and MAPK (specifically p38 or JNK) which induce expression of early response genes including proinflammatory cytokines $[1,2,4]$. On the other hand, TRIF adaptor leads to the activation of IRF3 that induces IFN- $\beta$ and interferon inducible genes. Pathway analyses of deregulated miRNAs highlight several cell signaling pathways that are integral to myeloid cellmediated innate responses. Our in silico analysis highlight MAPK, Ras, Raf1 signaling to be impacted by LPS responsive miRNAs. PI3K-Akt pathway/ phosphatidylinositol signaling was significantly impacted by differentially expressed miRNAs across all the datasets as predicted in our analysis. Akt- 1 has been demonstrated to play key role in endotoxin tolerance [22]. This protein regulates expression of various miRNAs including let-7e and miR-181c (positively) and miR-155 and miR-125b (negatively). Let-7e controls TLR response through targeting of TLR4 while, miR-155 regulates tolerance to immune response by targeting suppressor of cytokine signaling 1 (SOCS1). Evidently, Akt -/- mice fails to exhibit endotoxin resistance suggesting the key role of Akt pathway. This further confirms that macrophages respond to E. coli LPS challenge by altering expression of miRNAs that regulate both MyD-dependent and independent signaling cascades and can control signaling cascades leading to their own expression through feedback mechanisms.

Innate immunity constitutes the first line of defense by which the host recognizes and responds to invading pathogens or their conserved molecular patterns. Indeed, defective TLR signaling has been associated with plethora of pathophysiological conditions and diseases. Single nucleotide polymorphisms (SNPs) in TLR2 and TLR4 have been associated with childhood asthma, chronic obstructive pulmonary disease (COPD) and colorectal cancer [30-32]. Increased TLR4 expression leads to type 2 diabetes by reducing insulin secretion by $\beta$-cells and together with TLR2 and TLR9 contribute to the development of neurological disorders like schizophrenia and autism $[32,33]$. These studies highlight importance of TLRs in immune response and maintaining human physiology.

In summary, our results demonstrate that primary human macrophages respond to the TLR4 agonist, E. coli LPS by modulating expression of various miRNAs. These miRNA profiles are responsive to LPS dose and incubation time. In turn, these miRNAs are predicted to target key genes linked to immunity, inflammation and pain. This indicates that miRNAs play an important role in shaping innate immune responses by $\mathrm{M} \varphi$.

\section{Acknowledgements}

The authors wish to thank Dr Yan Shi and Ms Yan Li for technical support. This work was supported by the School of Dentistry, University of North CarolinaChapel Hill, the American Association of Endodontists Foundation, and the $\mathrm{NIH} /$ NIDCR [R01DE021052]

\section{References}

1. Mogensen TH (2009) Pathogen recognition and inflammatory signaling in innate immune defenses. Clin Microbiol Rev 22: 240-27, Table of Contents.

2. Newton K, Dixit VM (2012) Signaling in innate immunity and inflammation. Cold Spring Harb Perspect Biol 4.

3. Bryant CE, Gay NJ, Heymans S,, Sacre S5, et al. (2015) Advances in Toll-like receptor biology: Modes of activation by diverse stimuli. Crit Rev Biochem Mol Biol 50: 359-379. 
Citation: Naqvi AR, Zhong S, Dang H, Fordham JB, Nares S, et al. (2016) Expression Profiling of LPS Responsive miRNA in Primary Human Macrophages. J Microb Biochem Technol 8: 136-143. doi: 10.4172/1948-5948.1000276

4. Takeda K, Akira S (2015) Toll-like receptors. Curr. Protoc .Immunol 109: 14-25.

5. Gay NJ, Symmons MF, Gangloff M, Bryant CE2 (2014) Assembly and localization of Toll-like receptor signalling complexes. Nat Rev Immunol 14 $546-558$

6. Ambros $\vee(2004)$ The functions of animal microRNAs. Nature 431: 350-355.

7. Bartel DP (2009) MicroRNAs: target recognition and regulatory functions. Cell 136: 215-233.

8. Naqvi AR, Islam MN, Choudhury NR, Haq QM (2009) The fascinating world of RNA interference. Int J Biol Sci 5: 97-117.

9. Lindsay MA (2008) microRNAs and the immune response. Trends Immuno 29: 343-351.

10. Sonkoly E, Ståhle M, Pivarcsi A (2008) MicroRNAs and immunity: novel players in the regulation of normal immune function and inflammation. Semin Cance Biol 18: 131-140.

11. Curtale G, Citarella F, Carissimi C, Goldoni M, Carucci N, et al. (2010) An emerging player in the adaptive immune response: microRNA-146a is a modulator of IL-2 expression and activation-induced cell death in T lymphocytes. Blood 115: 265-273.

12. Taganov KD, Boldin MP, Chang KJ, Baltimore D (2006) NF-kappaB-dependen induction of microRNA miR-146, an inhibitor targeted to signaling proteins of innate immune responses. Proc Natl Acad Sci U S A 103: 12481-12486.

13. Chen XM, Splinter PL, O'Hara SP, LaRusso NF (2007) A cellular micro-RNA let-7i, regulates Toll-like receptor 4 expression and contributes to cholangiocyte immune responses against Cryptosporidium parvum infection. J Biol Chem 282: $28929-28938$

14. Benakanakere MR, Li Q, Eskan MA, Singh AV, Zhao J, et al. (2009) Modulation of TLR2 protein expression by miR-105 in human oral keratinocytes. J Biol Chem 284: 23107-23115.

15. Santaolalla R, Abreu MT (2012) Innate immunity in the small intestine. Curr Opin Gastroenterol 28: 124-129.

16. Payton JE, Grieselhuber NR, Chang LW, Murakami M, Geiss GK, et al. (2009) High throughput digital quantification of mRNA abundance in primary human acute myeloid leukemia samples. J Clin Invest 119: 1714-1726.

17. Malkov VA, Serikawa KA, Balantac N, Watters J, Geiss G, et al. (2009) Multiplexed measurements of gene signatures in different analyses using the Nanostring nCounter Assay System. BMC Res 2: 80

18. Xu Z, Zhou A, Ni J, Zhang Q, Wang Y, et al. (2015) Differential expression of miRNAs and their relation to active tuberculosis. Tuberculosis (Edinb) 95 395-403.

19. Costa-Reis P, Russo PA, Zhang Z, Colonna L, Maurer K (2015) The role of miRNAs and HER2 in proliferative lupus nephritis. Arthritis Rheumatol. 67 2415-2426.
20. Naqvi AR, Fordham JB, Khan A, Nares S (2014) MicroRNAs responsive to Aggregatibacter actinomycetemcomitans and Porphyromonas gingivalis LPS modulate expression of genes regulating innate immunity in human macrophages. Innate Immun 20: 540-551.

21. Androulidaki A, lliopoulos D, Arranz A, Doxaki C, Schworer S, et al. (2009) The kinase Akt1 controls macrophage response to lipopolysaccharide by regulating microRNAs. Immunity 31: 220-231.

22. Tserel L, Runnel T, Kisand K, Pihlap M, Bakhoff L (2011) MicroRNA expression profiles of human blood monocyte-derived dendritic cells and macrophages reveal miR-511 as putative positive regulator of Toll-like receptor 4 . J Biol Chem 286: 26487-26495.

23. Nares S, Moutsopoulos NM, Angelov N, Rangel ZG, Munson PJ, et al. (2009) Rapid myeloid cell transcriptional and proteomic responses to periodontopathogenic Porphyromonas gingivalis. Am J Pathol 174: 1400-1414

24. Naqvi AR, Fordham JB, Nares S (2015) miR-2, miR-30b, and miR-142-3p regulate phagocytosis in myeloid inflammatory cells. J Immunol 194: 19161927.

25. Tengku-Muhamma TS, Hughes TR, Cryer A, Ramji DP (1999) Synergism between lipopolysaccharide and interferon gamma in the regulation of lipoprotein lipase in macrophages. Cytokine 11: 408-415.

26. Guha M, Mackman N (2002) The phosphatidylinositol 3-kinase-Akt pathway limits lipopolysaccharide activation of signaling pathways and expression of inflammatory mediators in human monocytic cells. J Biol Chem 277: 32124 32132.

27. Luyendyk JP, Schabbauer GA, Tencati M, Holscher T, Pawlinski R (2008) Genetic analysis of the role of the PI3K-Akt pathway in lipopolysaccharideinduced cytokine and tissue factor gene expression in monocytes/macrophages. J Immunol 180: 4218-4226.

28. Laird MH, Rhee SH, Perkins DJ, Medvedev AE (2009) TLR4/MyD88/PI3K interactions regulate TLR4 signaling. J Leukoc Biol 85: 966-977.

29. Kerkhof M, Postma DS, Brunekreef B, Reijmerink NE, Wijga AH, et al. (2010) Toll-like receptor 2 and 4 genes influence susceptibility to adverse effects of traffic-related air pollution on childhood asthma. Thorax 65: 690-697.

30. Budulac SE, Boezen HM, Hiemstra PS, Lapperre TS, Vonk JM, et al. (2012) Toll-like receptor (TLR2 and TLR4) polymorphisms and chronic obstructive pulmonary disease. PLoS One 7: e43124.

31. Pimentel-Nunes P, Teixeira AL, Pereira C, Gomes M (2013) Functiona polymorphisms of Toll-like receptors 2 and 4 alter the risk for colorectal carcinoma in Europeans. Dig Liver Dis 45: 63-69.

32. Ladefoged M, Buschard K, Hansen AM (2013) Increased expression of toll-like receptor 4 and inflammatory cytokines, interleukin- 6 in particular, in islets from a mouse model of obesity and type 2 diabetes. APMIS 121: 531-538.

33. Enstrom AM, Onore CE, Van de Water JA, Ashwood P (2010) Differentia monocyte responses to TLR ligands in children with autism spectrum disorders. Brain Behav Immun 24: 64-71. 